\title{
Efecto antifúngico de hidrodestilados de Zingiber officinale Roscoe sobre Moniliophthora roreri (Cif\&Par)
}

\section{Hidrodestillates antifungal effect from Zingiber officinale Roscoe on Moniliophthora roreri (Cif \& Par)}

Fecha de recepción: 17 de marzo de 2015 Fecha de aprobación: 3 de junio de 2015
José Gregorio Joya-Dávila1, Sandra Isabel Ramírez-González ${ }^{2}$ Orlando López-Báez ${ }^{3}$ Álvaro Enrique Alvarado-Gaona ${ }^{4}$

\section{Resumen}

La moniliasis, ocasionada por el hongo Moniliophthora roreri (Cif. \& Par), es la principal enfermedad del cacao en Latinoamérica, y las medidas para su manejo sostenible son aún escasas. Con base en reportes previos, el objetivo de este trabajo fue optimizar el proceso de extracción por destilación de Zingiber officinale, evaluando in vitro su efecto antifúngico sobre conidias de $M$. roreri. Se elaboraron ocho hidrodestilados con material fresco (300 y $600 \mathrm{~g} / \mathrm{L}$ ) y seco al sol (45 y $90 \mathrm{~g} / \mathrm{L}$ ), con dos solventes (agua-alcohol, relación 10:1 y 10:0), y en tubos de ensayo con conidias del hongo, mediante la técnica de cultivo en medio líquido (agua-extracto de cacao), se adicionó cada hidrodestilado de Z. officinale en relación 1:1 $\mathrm{v} / \mathrm{v}$, determinando su efecto sobre la formación y germinación de conidias del hongo en cinco momentos de observación $(0,24,48,72$ y 96 horas). Los resultados muestran que todos los hidrodestilados de Z. officinale presentan metabolitos con efectos de inhibición sobre las variables evaluadas, siendo el tratamiento J5 (Z. officinale seco, 45 g/L, relación 10:1 agua: alcohol) el mejor, pues alcanza el 88\% y el $100 \%$ de control sobre la formación y germinación de conidias, respectivamente, con respecto al testigo absoluto a las 72 horas, siendo posible optimizar la extracción mediante destilación utilizando $45 \mathrm{~g} / \mathrm{L}$ de material seco y el uso de solvente agua-alcohol en una relación 10:1.

Palabras clave: Antifúngicos, Zingiber officinale, extractos vegetales, jengibre, monilia, Theobroma cacao, Moniliophthora roreri, Conidias de hongo.

1 Profesional independiente (Tunja-Boyacá, Colombia).

2 Ph.D. Universidad Autónoma de Chiapas (Tuxtla Gutiérrez-Chiapas, México). sandra.ramirez@unach.mx.

3 Ph.D. Universidad Autónoma de Chiapas (Tuxtla Gutiérrez-Chiapas, México). olopez@unach.mx.

4 M.Sc. Universidad Pedagógica y Tecnológica de Colombia (Tunja-Boyacá, Colombia). alvaro.alvarado@uptc.edu.co. 


\begin{abstract}
The frosty pod rot caused by the fungus Moniliophthora roreri (Cif. \& Par) is the main disease of cocoa in Latin America, while the measures for their sustainable management are still limited. Based on previous reports the aim of this study, was to optimize the extraction process by the distillation of Zingiber officinale, and evaluating in vitro their antifungal effect on $M$. roreri. Eight hidrodestilleted were prepared with fresh material (300 and $600 \mathrm{~g} / \mathrm{L}$ ) and sun dried (45 and $90 \mathrm{~g} / \mathrm{L}$ ), with two solvents (Water:Alcohol ratio 10:1 and 10:0) for which the cultivation technique used, was test tubes in liquid medium (water extracted from cocoa ) with conidia fungus. It was added each hidrodestillated of Z. officinale in ratio (1:1 v/v), by which determines its effect on the formation and germination of the fungus conidia in five observation times $(0,24,48,72$ and 96 hours).

The results indicate that all hidrodestillators of the Z. officinale show metabolites with inhibitory effects on the variables evaluated, with the J5 (dried Z. officinale, $45 \mathrm{~g} / \mathrm{L}$ ratio 10:1 water: alcohol) being the best treatment, due that it reaches from $88 \%$ to $100 \%$ control over the Conidias formation and germination respectively, with regard to the absolute witness at 72 hours, being also possible to optimize the distillation extraction by using $45 \mathrm{~g} / \mathrm{L}$ dry material and employ solvent water:alcohol in a 10:1 relationship.
\end{abstract}

Keywords: Antifungal, Zingiber officinale, Plant Extracts, Ginger, Frosty Pod Rot, Theobroma cacao, Moniliophthora roreri, Conidia fungus. 


\section{Introducción}

La moniliasis del cacao, causada por el hongo Moniliophthora roreri (Cif \& Par), afecta exclusivamente al fruto en cualquier etapa de desarrollo; entre sus síntomas se encuentran protuberancias, puntos aceitosos, amarillamiento o maduración prematura y manchas chocolate o necróticas $(1,2$, $3,4)$; ocasiona la pérdida total de sus semillas o disminución en su calidad organoléptica, convirtiéndose en el principal problema en 11 países del continente americano, en los que están México y Colombia $(5,6)$. M. roreri tiene su centro de origen en Colombia $(7,8,9,10)$; a la fecha se han encontrado cinco grupos genéticos del hongo $(11,12)$. En México fue detectada por primera vez en 2005, en el municipio de Pichucalco, Chiapas (4), ocasionando el derribo y abandono de plantaciones, bajas considerables de la producción, empobrecimiento de los productores y deterioro ambiental $(13,14)$. En Colombia, M. roreri es devastador y las estrategias de control tradicional han generado resultados colaterales, como la resistencia a fungicidas y mutaciones que han originado varias cepas en algunas regiones $(15,16)$. Los fungicidas de síntesis química para el control de M. roreri han sido ensayados en diversos lugares, pero sus resultados no son del todo efectivos para el manejo de esta enfermedad, $y$, en cambio, ocasionan contaminación y, a menudo, resulta antieconómico $(17,18$, $1,19,20,21,22,23)$.

Los extractos vegetales son una herramienta útil para el control de plagas y enfermedades, tienen un potencial muy alto para manejar los principales problemas fitosanitarios de la producción agrícola $(24,25)$. Como parte de su metabolismo, las plantas sintetizan componentes que se conocen como metabolitos secundarios, cuyas propiedades químicas se han investigado ampliamente desde mediados del siglo XIX $(26,27)$. Investigaciones realizadas en México muestran la gran diversidad de plantas que presentan metabolitos capaces de inhibir el crecimiento y desarrollo de patógenos (Phytophthora spp., Colletrotrichum gloeosporioides, Moniliophthora roreri) $(28,29)$, tales como orégano (Origanum vulgare L), jengibre (Zingiber officinale Roscoe) y maguey morado (Tradescantia spathacea Swartz). El jengibre ha mostrado efecto sobre la inhibición de diversos tipos de bacterias y hongos que causan enfermedades en animales y en plantas de cultivo, tanto a nivel de campo como en la poscosecha $(30,31,32,33,34,35,36,25)$.

Teniendo en cuenta la necesidad de buscar nuevas alternativas que sean amigables con los agroecosistemas y eficaces para el control de esta enfermedad tan limitante, pero accesibles a los productores de cacao, y considerando los reportes de Ramírez (14), se realizó esta investigación con el objetivo de optimizar el proceso de extracción por destilación de Z. officinale Roscoe, evaluando su capacidad antifúngica sobre la formación y germinación de conidias de M. roreri (Cif \& Par) aislado de mazorcas de cacao en plantaciones de México.

\section{Materiales y métodos}

Aislamiento del patógeno: El hongo $M$. roreri fue multiplicado a partir de una cepa previamente aislada de muestras de frutos enfermos, del municipio de Comalcalco, Tabasco, México; aislada según metodología descrita por Ramírez (14), en el laboratorio de Agrotecnologías de la AUDES Cacao-Chocolate, de la Universidad Autónoma de Chiapas.

Obtención de los hidrodestilados: Fueron elaborados a partir de jengibre (Z. officinale Roscoe) en un destilador adaptado para la obtención de hidrodestilados según metodología descrita por Ramírez (14), en el Laboratorio de Agrotecnologías de la AUDES Cacao-Chocolate; para su preparación se consideró utilizar dos diferentes cantidades de material vegetal (tanto fresco como seco al sol), así como dos tipos de solventes (agua:alcohol, relación 10:1, y agua). Se obtuvieron ocho hidrodestilados, que se describen en la Tabla I. Para el proceso de extracción se colocó el material vegetal y el solvente respectivo en la marmita, y se sometió a calentamiento constante, hasta obtener el hidrodestilado. 
Tabla I. Convenciones para los diferentes tratamientos.

\begin{tabular}{|c|c|c|c|c|}
\hline \multicolumn{2}{|c|}{ Tratamientos. } & Planta & Cantidad & Relación \\
\hline 1 & $J_{1}$ & \multirow{4}{*}{ Jengibre fresco } & 300 & $10: 1$ \\
\hline 2 & $\mathrm{~J}_{2}$ & & 600 & $10: 1$ \\
\hline 3 & $\mathrm{~J}_{3}$ & & 300 & 10:0 \\
\hline 4 & $\mathrm{~J}_{4}$ & & 600 & 10:0 \\
\hline 5 & $\mathrm{~J}_{5}$ & \multirow{4}{*}{ Jengibre seco } & 45 & $10: 1$ \\
\hline 6 & $\mathrm{~J}_{6}$ & & 90 & $10: 1$ \\
\hline 7 & $\mathrm{~J}_{7}$ & & 45 & 10:0 \\
\hline 8 & $\mathrm{~J}_{8}$ & & 90 & 10:0 \\
\hline 9 & \multicolumn{4}{|c|}{ Testigo absoluto (Test. Abs) Agua destilada } \\
\hline
\end{tabular}

Prueba en medio líquido en tubos de ensayo: Se utilizó la metodología descrita Ochoa (38), que permite determinar el número de conidias totales y el número de conidias germinadas en presencia de cada tratamiento.

Solución madre: Se tomaron cuatro cajas de Petri de $50 \mathrm{~mm}$ que contenían cultivo de $M$. roreri de 12 días de sembrado; se les realizó un raspado superficial del hongo y se lavó con $50 \mathrm{ml}$ de agua destilada estéril; se agregaron en un Erlenmeyer y se les añadió $50 \mathrm{ml}$ de extracto de cacao y una gota de tween 80 .

Solución con hidrodestilado: La solución madre se dividió en seis tubos de ensayo: tres como testigo (con $5 \mathrm{ml}$ de agua destilada $+5 \mathrm{ml}$ de solución madre) y tres que contenían el hidrolato por analizar $(5 \mathrm{ml}$ del hidrodestilado $+5 \mathrm{ml}$ de solución madre); luego se agitaron en vortex para homogeneizar y se realizaron las lecturas en la cámara de Neubauer, con tres repeticiones, contabilizando el número de conidias totales y el número de conidias germinadas, en cinco momentos de observación. Los tubos con los tratamientos y sus respectivos testigos se incubaron en la oscuridad a $28^{\circ} \mathrm{C}$ $+/-2^{\circ} \mathrm{C}$.

Variables evaluadas: Formación de conidias totales y número de conidias germinadas a 0, 24, 48, 72 y 96 horas, según metodología descrita por Ramírez (37) y Ochoa (38).

Diseño experimental: Se empleó un diseño completamente al azar, con nueve tratamientos y tres repeticiones por tratamiento a las 0, 24, 48, 72 y 96 horas. Se realizó un análisis de varianza, y cuando se detectaron diferencias significativas se aplicó la prueba de comparación de medias de Tukey al 5\%, empleando el programa estadístico SPSS STATISTICS 2.0.

\section{Resultados y discusión}

De acuerdo con el análisis estadístico, se observan diferencias significativas en el efecto de los tratamientos sobre la formación de conidias a las $0,24,48,72$ y 96 horas (Figura 1). A las 0 horas, el tratamiento con mayor formación de conidias correspondió al testigo absoluto (agua destilada), con $258,33 \times 10^{4}$ conidias $\mathrm{ml}^{-1}$, y el tratamiento que presentó menor formación de estas estructuras fue $\mathrm{J} 5$, con $58,3 \times 10^{4}$ conidias $\mathrm{ml}^{-}$ 1 , mostrando diferencias estadísticas con respecto al testigo absoluto, pero no con los tratamientos J1, J4, J6, J7 y J8 (Figura 1A), los cuales registraron valores mayores entre los 62,5 al $70,83 \times 10^{4}$ conidias $\mathrm{ml}^{-1}$, presentando porcentajes de inhibición entre el $77,42 \%$ y el $72.58 \%$ para estos tratamientos.

A las 24 horas, el tratamiento con mayor formación de conidias fue el testigo absoluto, mostrando diferencias estadísticas significativas con los demás tratamientos, siendo el tratamiento J5, con $44,4 \times 10^{4}$ conidias $\mathrm{ml}^{-1}$, 
el que inhibió en mayor grado su formación, con un $74 \%$; sin embargo, todos los extractos inhibieron la formación de conidias, en comparación con el testigo (Figura 1B).

A las 48 horas, el tratamiento testigo presentó diferencias estadísticas significativas en relación con los demás tratamientos, siendo $\mathrm{J} 5$ y $\mathrm{J} 7$ los que presentaron la menor formación de conidias con $41,67 \times 10^{4}$ conidias $\mathrm{ml}^{-1}$, sin embargo, este tratamiento no registró diferencias estadísticas con los tratamientos $\mathrm{J} 6$ y J8, que fueron preparados a partir de material seco (Figura 1C) y lograron inhibir la formación de conidias en un rango del $81.76 \%$ al $79.33 \%$, con respecto al testigo absoluto. A esta hora, el testigo absoluto también se presentó germinación de conidias, con $3.47 \times 10^{4}$ conidias $\mathrm{ml}^{-1}$, mientras que los demás tratamientos inhibieron por completo la germinación de estas estructuras.

A las 72 horas, el testigo presentó la mayor germinación y formación de conidias, y registró diferencias estadísticas significativas con los demás tratamientos, continuando el tratamiento 5 con el mejor comportamiento en la inhibición en la formación de conidias, con $88.31 \%$, seguido por los tratamientos J6 y J8 (Figura 1D), que no registraron entre ellos diferencias estadísticas y tampoco permitieron la germinación de las conidias.

A las 96 horas, el tratamiento que mejor comportamiento presentó fue el J2, con $19,4 \times 10^{4}$ conidias $\mathrm{ml}^{-}$ ${ }^{1}$, presentando solamente diferencias significativas en cuanto al testigo (Figura 1E).

El tratamiento J5 presentó los valores más bajos de conidias totales en el transcurso del tiempo, llegando a inhibir un $88 \%$ la formación de conidias (valor registrado a las 72 horas de evaluación), resultado igual al que reporta Ramírez (37) con el extracto de corteza de canela a las 96 horas de evaluación. Estos resultados encontrados para Jengibre coinciden con los reportados por Nguefak et al. (31), quienes determinaron el efecto inhibitorio de $Z$. officinale sobre aislamientos de tres hongos procedentes de alimentos (F.moniliforme, A. flavus y A. fumigatus), extractos que, al igual, fueron obtenidos por destilación y que mostraron inhibición en el crecimiento y formación de conidias de los tres hongos, pero a concentraciones superiores que T. vulgaris. Además, el jengibre tiene un efecto inhibidor en su aceite a concentraciones altas, mostrando presencia de fenilpropanoides, compuestos que le dan propiedades de control sobre levaduras como C. albicans, R. glutinis, S. pombe, S. cerevisiae, Y. lypolitica (35).

Para la germinación de conidias se presentaron diferencias significativas entre tratamientos con el testigo absoluto a las 48, 72 y 96 horas de observación. A las 48 horas todos los tratamientos presentaron inhibición en la germinación de conidias del $100 \%$, mientras que el testigo presentó una germinación de $3,47 \times 10^{4}$ conidias $\mathrm{ml}^{-1}$. A las 96 horas el testigo presento $6,48 \times 10^{4}$ conidias $\mathrm{ml}^{-1}$, mientras que los demás tratamientos presentaron inhibición del $100 \%$. Los resultados muestran que los hidrolatos de jengibre, obtenidos tanto con material fresco como seco al sol, así como con solventes agua:alcohol y agua, permiten extraer metabolitos capaces de inhibir la germinación de conidias de $M$. roreri, evidenciando un alto potencial de los hidrodestilados obtenidos de jengibre en el manejo de este patógeno, ya que la inhibición en la germinación de esporas es un efecto muy deseable en los productos para el control de $M$. roreri, pues aunque las conidias del patógeno logren depositarse en la superficie del fruto, el contacto con el hidrolato de jengibre estaría impidiéndoles formar el tubo germinativo (38). 

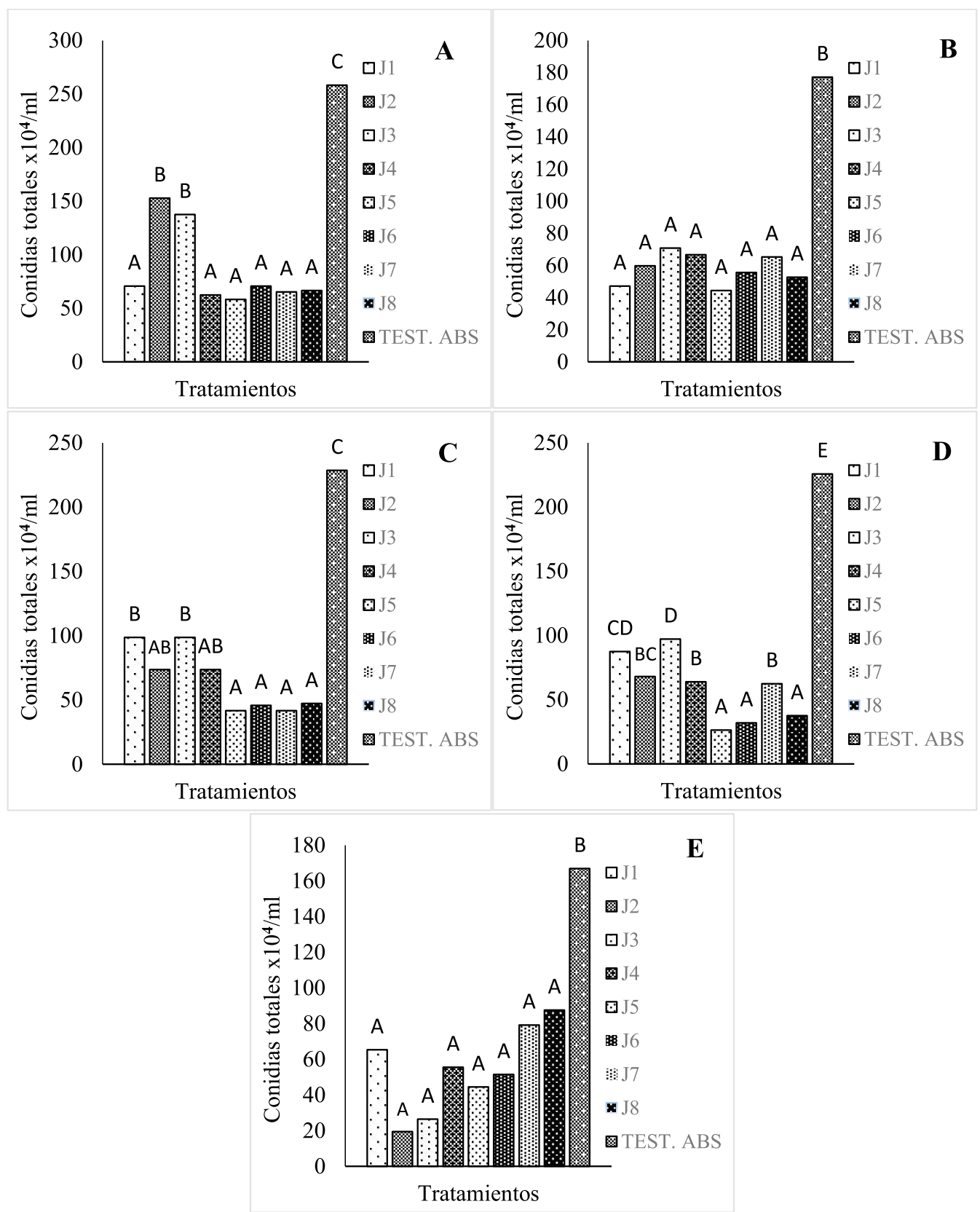

Figura 1. Efecto de los extractos de jengibre sobre el número de conidias totales $\times 10^{4} \mathrm{ml}^{-1}$. A) 0 horas, B) 24 Horas, C) 48 Horas, D) 72 horas, E: 96 Horas. Tratamientos con letras distintas indican diferencias significativas según la prueba de Tukey $(\mathrm{P} \leq 0.05)$.

Las conidias son los únicos propágulos infecciosos de $M$. roreri, y el hongo requiere germinar sobre la superficie del fruto para poder realizar la infección e iniciar con ello su proceso invasivo, de manera que, al inhibir la formación de conidias e impedir la germinación de estas estructuras, los eventos de infección serán reducidos y las probabilidades de 
infección y desarrollo de la enfermedad serán menores (5).

\section{Conclusiones}

El tratamiento J5 (jengibre seco, $45 \mathrm{~g} / \mathrm{L}$, relación 10:1 agua:alcohol) fue el mejor hidrodestilado de los ocho evaluados, el cual presentó los mejores niveles de control en la formación y germinación de conidias de $M$. roreri en el transcurso del tiempo; por lo cual constituye una alternativa potencial para el manejo de la moniliasis del cacao.

Es posible optimizar el proceso de obtención de hidrodestilado de jengibre utilizando material seco y el uso de solvente agua: alcohol en una relación 10:1, lo cual permite una mayor extracción de metabolitos activos que permiten controlar in vitro $M$. roreri.

\section{Referencias}

(1) Merchán, M. Avances en la investigación de la moniliasis del cacao en Colombia. In: La moniliasis del cacao. Enríquez, G. (ed.). Compendio de los trabajos presentados en el Seminario llevado a cabo en el CATIE. Informe Técnico No. 28. Centro Agronómico Tropical de investigación y ense-anza (CATIE), Costa Rica. 1980. Páginas 53-69.

(2) Evans, H. Invasive Neotropical Pathogens of Tree Crops. Tropical Mycology, 2002; 2(1): 98-105. DOI: http://dx.doi. org/10.1079/9780851995434.0083.

(3) Oliveira, M. y Luz, E. Identificação e manejo das principais doenças do cacaueiro no Brasil. Ilhéus, CEPLAC/ CEPEC/SEFIT. 132 p., 2005.

(4) López, O.; González, O.; Ramírez, S.; Lee, V.; Ramírez, M.; Alvarado A. \& Gehrke, M. Diagnóstico y técnicas para el manejo de la moniliasis del cacao. Universidad Autónoma de Chiapas; Universidad Pedagógica y Tecnológica de Colombia. Impreso: Digital. Tuxtla Gutiérrez, Chiapas, México. 40 p., 2006.
(5) Phillips, W.; Aime, C.; y Wilkinson, J. Biodiversity and biogeography of the cacao (Theobroma cacao) pathogen Moniliophthora roreri in tropical America. Plant Pathol. 2007; (56): 911-922. DOI: http://dx.doi.org/10.1111/ j.1365-3059.2007.01646.x.

(6) Sánchez, F. y Garcés F. Moniliophthora roreri (Cif y Par) Evans et ál., en el cultivo de cacao. Scientia Agropecuaria. 2012; 3(3): 249-258. DOI: http://dx.doi.org/10.17268/sci.agropecu.2012.03.06.

(7) Phillips. W.; Castillo, J.; Krauss, U.; Rodriguez, E.; Wilkinson, J. Evaluation of cacao (Theobroma cacao) clones against seven Colombian isolates of Moniliophthora roreri from four pathogen genetic groups. Plant Pathology. 2005; (54): 483-490. DOI: http://dx.doi.org/10.1111/j.1365-3059.2005.01210.x.

(8) Phillips, W. Origen, biogeografía, diversidad genética y afinidades taxonómicas del hongo Moniliophthora roreri (Cif) Evans et al., del cacao (Theobroma cacao L.) determinadas mediante evidencia molecular, fitopatológica y morfofisiológica. 2006. Recuperado el 23 de agosto de 2015. Disponible en: www. Catie. ac.cr.

(9) Grisales, S. y Afanador, L. Análisis de variabilidad genética en Moniliophthora roreri con AP-PCR y RAPD en Antioquia, Colombia. Revista Colombiana de Biotecnologia. 2007; 9(2). 15-32.

(10) Jaimes, Y. y Aranzazu, F. Manejo de las enfermedades del cacao (Theobroma cacao L.) en Colombia, con énfasis en Monilia (Moniliophthora roreri). In: Hoyos L.M. (ed.). Corporación Colombiana de Investigación Agropecuaria, Corpoica, Colombia. p. 90. 2010.

(11) Phillips, W.; Aime, M. The causal agents of 'witches' broom and frosty pod rot of cacao chocolate (Theobroma cacao) form a new lineage of Maras miaceae. Mycologia. 2005; (97): 1012-1022. 
(12) Álvarez, J.; Martínez, S. y Coy J. Estado de la Moniliasis del cacao causada por Moniliophthora roreri en Colombia. Acta agronómica. 2014; 63(4). 388-399. DOI: http://dx.doi. org/10.15446/acag.v63n4.42747.

(13) Ramírez, G, Sandra. La moniliasis un desafío para lograr la sostenibilidad del sistema cacao en México. Tecnología en Marcha. 2008a; 21(1): 97-110.

(14) Ramírez, S.; López O.; Guzmán T.; Munguía S.; Espinosa, S. Actividad Antifúngica in vitro de extractos de Oreganum. vulgare L., Tradescantia. spathacea Swartz y Zingiber officinale Roscoe sobre Moniliophthora roreri (Cif\& Par). Tecnología en Marcha. 2011a; 24(2): 3-17

(15) Meinhardt, L. y Rincones, J. Moniliophthora perniciosa, the causal agent of witches' broomdisease of cacao: what's new from this old foe? Mol. Plant. Pathol, 2008; 9(5): 577588. DOI: http://dx.doi.org/10.1111/j.13643703.2008.00496.x.

(16) Meinhardt, W.; Costa, G.; Thomazella, P.; Teixeira, J. Genome and secretome analysis of the hemibio trophic fungal pathogen, Moniliophthora roreri, which causes frosty pod rot disease of cacao: mechanisms of the biotrophic and necrotrophic phases. BMC Genomics, 2014; 15(1): 164-176. DOI: http:// dx.doi.org/10.1186/1471-2164-15-164.

(17) Meza, C. y León, V. "Control químico de la moniliasis y mancha de agua del cacao". Revista de la Facultad de Agronomía (Venezuela), 1972; 2(1): 17-29.

(18) Suárez, C. Las enfermedades del cacao en Latinoamérica. In: $7^{\circ}$ Conferencia Internacional de Investigación en Cacao. Douala, Cameroun. $1979 ; 251-254$.

(19) Achicanoy, H. \& Buritica, P. Evaluación in vitro de fungicidas para el control de Crinipellis perniciosa (Stahel) Singer y Moniliophthora roreri (Cif \&Par) Evans et ál., In: $8^{\text {a }}$. Conferencia Internacional de Investigación en Cacao. Cartagena, Colombia. Proceedings: 1981; 419-423.

(20) González, L.C. Epifitiología y combate de la moniliasis del cacao. Universidad de Costa Rica. Facultad de Agronomía, San Pedro De Montes de Oca, Costa Rica. Informe anual de proyecto de investigación. 1982; 21 p.

(21) Cruz, A. Evaluación de la remoción de frutos, la aplicación de fungicidas y la polinización artificial sobre la incidencia de moniliasis y la producción de cacao. Tesis para ingeniero agrónomo. Escuela de Fitotecnia, Facultad de Agronomía, Universidad Nacional de Costa Rica. 1986.

(22) Martí, J.; Galindo, J.; Ramírez, C. \& Enríquez, G. Evaluación del combate biológico y químico de la moniliasis (Moniliophthora roreri) del cacao en Costa Rica. In: 10ª . Conferencia Internacional de Investigación en Cacao. Santo Domingo, República Dominicana. Proceedings: 1987 ; 453-497.

(23) Sánchez, F.; Gamboa, E. \& Rincón, J. Control químico y cultural de la Moniliasis (Moniliophthora roreri cif \& par) del cacao (Theobroma cacao L) en el Estado Barinas. Rev. Fac. Agron. 2003; 20(1): 188-194.

(24) Hernández, A.; Bautista, S; Velázquez, M. Prospectiva extracto vegetales para controlar enfermedades en Poscosecha. Rev. Fitc. Mex. 2007; 30(2):119-123.

(25) Barrera, L. \& Bautista, S. Actividad antifúngica de polvos, extractos y fracciones de Cestrum nocturnum L. sobre el crecimiento micelial de Rhizopus stolonifer Vuill. Revista Mexicana de Fitopatología. 2008; 26(1):27-31.

(26) Vergara, R. De la agricultura tradicional a la agricultura biológica. Memorias Seminario Regional. Universidad Pedagógica y Tecnológica de Colombia. 1997. 
(27) Croteau, R.; Kutchan, M.; Lewis, G. Natural products (secondary metabolites). In Biochemistry and Molecular Biology of Plants. Buchanan, B.B.; Gruissem, W.; Jones, R.L. (Editors). American Society of Plant Physiologists. Rockville, US. p. 2000; 1250-1318.

(28) Ramírez, S.; López O. El manejo orgánico integral de insectos plagas y enfermedades en el cultivo del cacao (Theobroma cacao L.). In: López B. O., Ramírez G. S. I., Ramírez G. M., Moreno B. G., Alvarado G. A. (Ed). (2006). Agroecología y agricultura orgánica en el trópico. Tunja: Editorial UPTC-UNACH, Tunja, Boyacá, Colombia. 2006.

(29) Ramírez, S. Extractos vegetales para el manejo orgánico de la mancha negra (Phytophthora palmivora) del cacao (Theobroma cacao). Tesis Maestría en Biotecnología. Facultad de Ciencias Químicas. Universidad Autónoma de Chiapas. Tapachula, Chiapas- México. 2008b.

(30) Bertelli D; M. Plessi \& F. Miglietta. Effect of microwaves on volatile compounds in origanum. Lebensm.-Wiss. u.-Technol. 2003; 36(1): 555-560.

(31) Nguefack, J.; Leth, V.; Amvam, P.; Mathur, B. Evaluation of five essential oils from aromatic plants of Cameroon for controlling food spoilage and mycotoxin producing fungi. International Journal of Food Microbiology. 2004; 94 (2): 329- 334. DOI: http://dx.doi.org/10.1016/j.ijfoodmicro.2004.02.017.

(32) Kulisic T.; Radonic, A.; Katalinic, V. \& Milos, M. Use of different methods for testing antioxidative activity of oregano essential oil. Food chemistry. 2004; 85: 633-640, 2004. DOI: http://dx.doi.org/10.1016/j.foodchem.2003.07.024.

(33) Nostro, A.; Blanco, R.; Cannatelli, A.; Enea, V.; Flamini, G.; Morelli, I. et al. Susceptibi- lity of methicillinresistant taphylococci to oregano essential oil, carvacrol and thymol. FEMS Microbiology Letters. 2004; 230: 191195. DOI: http://dx.doi.org/10.1016/S03781097(03)00890-5.

(34) Sahin, M.; Güllüce, D; Sökmen, M.; Polissiou, $\mathrm{G}$; Özer, H. Biological activities of the essential oils and methanol extract of Origanum vulgare ssp. vulgare in the Eastern Anatolia region of Turkey. Food Control. 2004; 15: 549-557. DOI: http://dx.doi.org/10.1016/j. foodcont.2003.08.009.

(35) Sacchetti, G.; Maietti, S.; Muzzoli, M.; Scaglianti, M.; Manfredini, S.; Radice, M. \& Bruni, R. Comparative evaluation of 11 essential oils of different origin as functional antioxidants, antiradicals and antimicrobials in foods. Food Chemistry. 2005; (91): 621-632. DOI: http:// dx.doi.org/10.1016/j.foodchem.2004.06.031.

(36) Hersch, B.; Lea-os, F. Antibacterial effects of commercial essential oils over locally prevalent pathogenic strains. México. Fitoterapia. 2005; (76): 453-457. DOI: http://dx.doi.org/10.1016/j.fitote.2005.03.006.

(37) Ramírez, S. Efectividad de extractos vegetales en el manejo de la Moniliasis (Moniliophthora roreri) del cacao (Theobroma cacao L.) en México. Tesis de Doctorado en Ciencias Naturales para el Desarrollo. Universidad Nacional. Heredia, Costa Rica. 2013.

(38) Ochoa, L. Preparados minerales en el manejo orgánico de la Moniliasis (Moniliophthora roreri (Cif. y Par.) Evans et al.) de Theobroma cacao L. En el municipio de Tecpatán, CHIAPAS - MÉXICO Tesis de Maestría, Ciencias en Producción Agropecuaria Tropical, Universidad Autónoma de Chiapas Facultad de Ciencias Agrícolas Campus IV. 2015. 